\title{
"Charge while driving" for electric vehicles: road traffic modeling and energy assessment
}

\author{
Francesco Paolo DEFLORIO ( $\square$ ), Luca CASTELLO, \\ Ivano PINNA, Paolo GUGLIELMI
}

\begin{abstract}
The aim of this research study is to present a method for analyzing the performance of the wireless inductive charge-while-driving (CWD) electric vehicles, from both traffic and energy points of view. To accurately quantify the electric power required from an energy supplier for the proper management of the charging system, a traffic simulation model is implemented. This model is based on a mesoscopic approach, and it is applied to a freight distribution scenario. Lane changing and positioning are managed according to a cooperative system among vehicles and supported by advanced driver assistance systems (ADAS). From the energy point of view, the analyses indicate that the traffic may have the following effects on the energy of the system: in a low traffic level scenario, the maximum power that should be supplied for the entire road is simulated at approximately $9 \mathrm{MW}$; and in a high level traffic scenario with lower average speeds, the maximum power required by the vehicles in the charging lane increases by more than $50 \%$.
\end{abstract}

Keywords Wireless charging, Cooperative driving, Traffic simulation, Mesoscopic, Energy estimation

\section{Introduction}

Electric vehicles that provide zero local emissions and high energy efficiencies are becoming a real alternative for

CrossCheck date: 16 January 2015

Received: 30 September 2014/ Accepted: 3 March 2015/Published online: 18 March 2015

(C) The Author(s) 2015. This article is published with open access at Springerlink.com

F. P. DEFLORIO, L. CASTELLO, I. PINNA, P. GUGLIELMI, Politecnico di Torino, 10129 Turin, Italy

$(\bowtie)$ e-mail: francesco.deflorio@polito.it future motorized mobility. However, their acceptance in the market is limited by the following disadvantages when compared with diffused classical internal combustion engine vehicles: autonomy, lack of recharging infrastructures with public access, the time consuming charging process, limited battery life, battery cost and compliant masses. Charge-while-driving (CWD) technology could represent an interesting opportunity to support the deployment of electric vehicles as a possible solution.

The majority of fully electric vehicles (FEVs) currently satisfy the electric energy requirements for motion with an on-board battery. Reference [1] analyzed the problems related to battery charging management, the uncertainty surrounding the monitoring of the state of charge (SOC), the limited availability of charging infrastructure and the long time required to recharge; problems that have generated range anxiety. Extensive research has claimed that the challenges of battery inefficiency and the large and wasted space in the FEVs can be overcome by the wireless power transfer (WPT) technology. This technology electrically conducts energy from a source to an electric device without any interconnecting mediums [2]. The maglev system, developed in the late 1970s, utilises the high speed of a travelling vehicle to generate electricity using a linear generator [3]. Reference [4] proposed a design methodology for loosely coupled inductive power transfer systems. Such systems were used for non-contact power transfer, normally, over large air-gaps to the moving loads. Reference [5] explored the integrated pricing of electricity and roads enabled with wireless power transfer technology. The on-line electric vehicle (OLEV) system [6] and its non-contact power transfer mechanism were developed by the Korea Advanced Institute of Science and Technology (KAIST) and presented in 2009. The OLEV is an electric transport system in which the vehicles absorb the power from power lines underneath the surface of the road. The 
aim of this research study is to present a method for analyzing the performance of the CWD system, from both traffic and energy points of view. Beginning with an electric vehicle supply equipment (EVSE) layout defined and analyzed in a previous study [7] and using the system requirements defined in the eCo-FEV project [8], a model for the traffic flow simulation is implemented to quantify and describe the time-dependent traffic parameters along the charging lane and the electric power that should be provided by an energy supplier for proper management of the charging system. The results of this analysis confirm the influence of different traffic conditions and system requirements on the quality of the charging service.

\section{Simulation model for the EVSE management}

The model developed in this paper could be applied to a freight distribution service. The FEV traffic flow simulated here represents a fleet of light vans that could be generated by, or directed to, a logistics centre for a distribution service. The fleet management in this case could include the CWD usage in the common route segment to allow vehicles to cover greater distances, avoiding wasted time for a stationary recharge and to control the mass of the batteries.

The analysis is applied to a $20 \mathrm{~km}$ roadway with multiple lanes scenario. The right-hand lane is reserved for the charging activities. In an actual road infrastructure example, this solution could be applied by allocating the slowest lane to CWD operations or by using the emergency lane with dynamic lane management. Figure 1 shows a CWD lane scheme, with two charging zones (CZs) represented. The EVSE includes inductive coils placed under the pavement surface, at a relative distance, which generate a high frequency alternating magnetic field to which the coil on the car couples and power is transferred to charge the battery.

A proper design procedure should consider both the service provider's need to minimize the installation and maintenance costs and the users' acceptance of the time required for a proper recharge in the CWD lane. Taking into account the results obtained in previous studies [7] performed for an electric light van, with a power provided per unit of length $(\mathrm{Pcz})$ of $50 \mathrm{~kW} / \mathrm{m}$ in the $\mathrm{CZs}$ and adopting a system efficiency $\eta_{\mathrm{s}}$ of $85 \%$ (from energy grid distribution to EV battery), the identified CWD system can be described by the following technical requirements: (1)

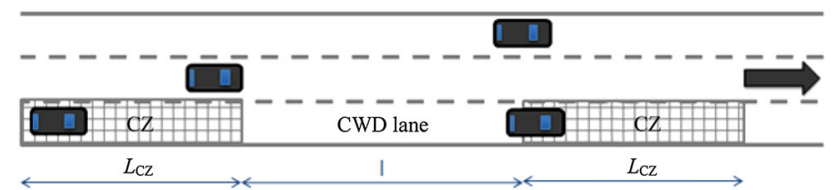

Fig. 1 Scenario layout for CWD in a road with three lanes
Length of the charging zones $\left(L_{\mathrm{CZ}}\right)=20 \mathrm{~m}$; (2) Inter-distance $(I)=30 \mathrm{~m}$; (3) Longitudinal dimension of the onboard charging device $\left(L_{\mathrm{CD}}\right)=1 \mathrm{~m}$.

In this layout, the energy equilibrium is possible at $60 \mathrm{~km} / \mathrm{h}$, whereas at lower speeds the SOC gain is positive. The two following operational speeds are defined for CWD: the highest speed $(60 \mathrm{~km} / \mathrm{h})$ should allow the vehicle to maintain its entering SOC, whereas the lowest speed $(30 \mathrm{~km} / \mathrm{h})$ should be a compromise between the recharge needs of vehicles with a low SOC and a minimum speed that can be accepted by the users. In this layout, by driving at the lowest speed, after $20 \mathrm{~km}$ in the CWD lane, the SOC increases by more than $7 \mathrm{kWh}$. This last case has been defined as "emer" status because this refers to a strategy applicable to emergency situations. The other charging vehicles have been classified with the "charge" status.

\subsection{Models for energy estimation}

In the CWD lane, a balance between the energy consumed for vehicle motion and the energy provided by the CZs should be established to monitor the SOC of the vehicle batteries during the observation period. The vehicle type included in the traffic flow is relevant because the mass and the aerodynamic parameters affect the energy consumption. After estimating the total average resistance force to motion $R_{\text {tot }}$ between two consecutive nodes, the average power consumed is calculated according to the following relationship, based on simple mechanical concepts:

$P_{\text {electric }}=\frac{R_{\mathrm{tot}} \cdot s}{\eta_{\mathrm{d}}}+P_{\mathrm{aux}}$

where $\eta_{\mathrm{d}}$ is the average driveline efficiency, which is assumed here constant for any average speed $s$ of the vehicle along the section; $P_{\text {aux }}$ is the auxiliary power that includes all consumption not related to the vehicle motion, such as the on-board electrical devices (e.g., lights and air conditioning). Finally, the energy consumed by the vehicle over time is obtained by multiplying the power consumed by the duration. In our scenarios, for sake of simplicity, the average slope will be assumed to equal zero.

The energy that the vehicle receives from the coils in the CWD lane $E_{\text {received }}$ can be calculated (as in (2)) by the electric power $(P)$ received by any $\mathrm{CZ}$, the number of $\mathrm{CZ}$ $n_{\mathrm{CZ}}$ and the occupancy time $t_{\mathrm{CZ}}$. This can then be related to the system element dimensions (CZs and on-board devicesLCD) and $P_{\mathrm{cz}}$, according to the following:

$$
\begin{aligned}
E_{\text {received }} & =P \cdot n_{\mathrm{CZ}} \cdot t_{\mathrm{CZ}} \\
& =\left(P_{\mathrm{CZ}} \cdot L_{\mathrm{CD}} \cdot \eta_{\mathrm{s}}\right) \cdot\left(\frac{L_{\text {roadsection }}}{L_{\mathrm{CZ}}+I}\right) \cdot\left(\frac{L_{\mathrm{CZeff}}}{s}\right)
\end{aligned}
$$

In (2), $L_{\text {CZeff }}$ is the CZ length in which the vehicles effectively recharge, considering the initial and final partial overlaps of the on-board device. When the vehicle crosses 
a transmitting coil, it receives the energy according to system efficiency $\eta_{\mathrm{s}}$ that depends on the distance between the coil(s) of the on-board device and the coil(s) of the $\mathrm{CZ}$ installed in the road pavement. Each CZ is subdivided into coils that are excited only if a receiving (and authorized) vehicle is above them. In this way, only the coils that are under the vehicle work, thus maintaining the emitted power inside a shielded zone, correspond to the vehicle occupancy.

\subsection{Traffic modelling}

The choice of the traffic modelling is derived from the specific requirements of the CWD system [8] as synthesized below.

1) It has been assumed as installed only along the righthand lane of the motorway because that lane is generally used by slower vehicles. Consequently, the model considers the lane disaggregation of traffic data.

2) The charging lane can be used for two different charging needs ("emer" or "charge") corresponding to two different vehicle speeds. Consequently, the model must consider different classes of vehicles.

One possible approach to effectively model this type of problem (multilane and multiclass) could be microsimulation, in which single vehicle trajectories are modelled with a small time step resolution and with their interaction on the road. An extensive review of traffic modelling approaches can be found in [9], whereas a microsimulation model application example is reported by [10]. Although the microsimulation approach meets the principal requirements of the traffic model for CWD, it does not model vehicle behavior according to their energy needs. The current SOC level of the vehicles and the fleet operators' eventual SOC target requirements influence drivers' decisions concerning lane changing, i.e., vehicles try to enter into the charging lane or to exit according to their needs. Therefore, specific rules must be defined and implemented to obtain realistic results from the traffic model. In addition, the detailed rules implemented in a micro-simulation model usually require an accurate calibration process, aimed at replicating the actual driving in traffic. However, the calibration process can be compromised in a CWD scenario whenever various ADAS are available on-board because they affect driving and traffic. Consequently, a mesoscopic approach would be more accurate than a microscopic one, because the latter is too detailed for this preliminary stage of CWD technology. Further comments on this issue will be reported in Sect. 3. A framework of mesoscopic traffic models can be found in [11], whereas a recent application of this type of model was proposed in [12].

The developed model represents single vehicle trajectories without introducing a detailed time resolution of the driving activities. It assumes that the CWD lane conditions can be described knowing only the data related to consecutive points. The point spacing, typically hundreds of meters, can be set based on the analysis required. For this reason, detailed traffic information has been updated only at these defined points, defined as "detection points" or "nodes", where it is interesting to know the time series of traffic parameters and the energy provided for the entire vehicle set detected in the related time period. The road segment between the consecutive nodes will be defined as "road section" or "section". Aggregated traffic information, such as average headways, delays and the number of overtake maneuvers, can be estimated along the CWD lane for any road section.

The logic scheme adopted for two consecutive nodes of the traffic model is depicted in Fig. 2.

In the traffic model, the arrival time of a vehicle at the node $i$ is first estimated based on its arrival time at the node $(i-1)$ and its desired speed. It is then adjusted, in a second step, according to the feasible headway for vehicles in the lane. Because of safety and possible technical reasons, headway less than a threshold value between two vehicles in the charging lane may not be allowed. If two vehicles detected at a certain node are too close, in terms of headway, the following one has to slow down until its headway is equal to the threshold.

The headway verification and correction is therefore performed only at discrete space steps, according to the mesoscopic modeling of traffic. In an actual scenario, it can be managed by drivers or by the cooperative system

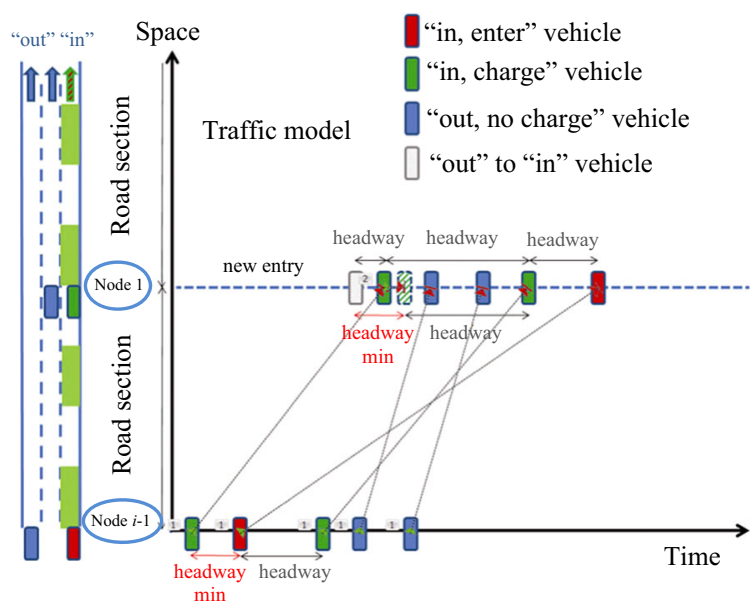

Fig. 2 Several trajectories in the time-space diagram to trace the arrival times of different vehicle types at consecutive nodes 
adapting the vehicle speed along the entire section before the node where the headway adjustment is performed.

The battery SOC, monitored along the road at each node, plays a crucial role because it influences drivers' decisions to use the CWD service or not. It is also the parameter used to divide the vehicles into different speed classes. In the model, the CWD lane entries are managed according to the following cooperative behavior: each vehicle requiring recharge moves into the CWD lane at the node, creating the necessary gap in the vehicle flow by slowing down the following vehicles. A block diagram reported in Fig. 3 describes the logic of the procedures and the various functions applied at every simulation step. More details on all the functions can be found in [7].

The proposed scenario refers to a freight distribution service. The decision to charge may be simplified because it depends not only on drivers and their final destinations, but primarily on the fleet operator. To restart the delivery operations in the second part of the day, all of the vehicles in the fleet may require an energy level adequate for their operation.

The analysis considers even the overtaking cases: a cooperative overtaking model at constant speed is implemented and the vehicle does not recharge while it is outside the charging lane. The traffic simulator has been implemented in Microsoft Excel platform using Visual Basic programming language, and more details on the model can be found in [13].

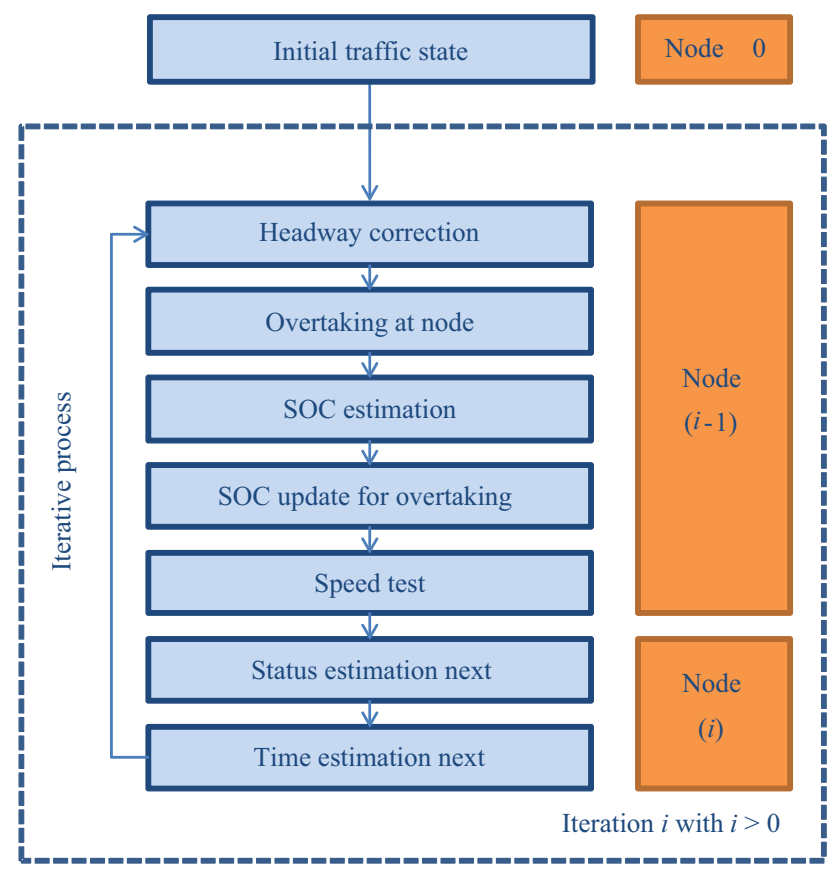

Fig. 3 Logic and procedures of the model

\section{Verification and validation process}

This chapter explains the model approach chosen, clarifying the reasons for the simplified assumptions and introducing a short discussion on verification and validation issues. Currently, the CWD system has been installed only in small test sites and, unfortunately, there are no opportunities to observe driver behavior in large-scale systems. Furthermore, even fully cooperative driving systems are not completely deployed. An actual traffic scenario, similar to that simulated, can be observed in long road tunnels in which vehicle spacing or headway greater than a predefined threshold should be maintained and all vehicles travel in a predefined speed range for safety reasons (e.g., the Mont Blanc tunnel).

Another important issue that should be considered is that the CWD technological environment will expand in the future. Therefore, it will involve another generation of vehicles, in which vehicle-to-vehicle communications will be used and many cooperative functions will be activated to facilitate the drive. In such a system, the observation of the current driving features is not relevant to model the traffic because vehicle motions and interactions depend more on the settings of the ADAS systems than on drivers' decisions.

For these reasons and considering the current stage of CWD technology development, calibration and validation operations based on empirical and on field observations are not possible. However, an extensive verification process can be performed by analyzing, testing and reviewing activities, according to the concepts defined in the ECSS standards [14]. In particular, a technical verification of the model response can be performed based on the following three consecutive test cases, each one aimed to verify different aspects: (1) single vehicle motion and the relationship between its behavior and its energy needs; (2) uniform vehicle flow without overtakes to verify if the model is able to correctly manage the headways between vehicles; (3) complex traffic interaction with overtaking maneuvers to assess the global interaction between vehicles, introducing overtaking maneuvers.

In the third stage of the model verification process, traffic results may be controlled by the following relevant parameters affecting traffic behavior.

1) Input traffic distribution (average headway, standard deviation and minimum value);

2) Vehicle speed for the two CWD classes (in the CWD lane where the speed is controlled and in the other lanes where the speed is derived from the densityspeed relationship);

3) Overtake management (duration, event detection, event activation, event recovery and multiple overtakes); 
4) Vehicle energy parameters (initial SOC, target SOC, SOC thresholds and energy consumption);

5) CWD parameters (system layout and power).

At this stage of the model development, the presented model has been validated by checking the satisfaction of the established technical requirements, based on the system engineering approach [15]. The main functional requirements for the model are the following: (1) the model shall estimate the number of vehicles in the CWD for any detection point; (2) the model shall consider possible random effects of input flow; (3) the model shall represent the traffic flow at any detection point and reveal if concentration of traffic and congestion occur along the lane; (4) the model shall take into account different values of the minimum headway allowed in the CWD to estimate possible effects on traffic and energy for the various CZs over time.

In the following sections, the model testing results are reported in an "ideal case", in which all of the subsystems and applications involved, such as the CWD booking and authorization functions, or the cooperative ADAS, which enables the vehicle cruise control or the cooperative overtaking, work properly. In this scenario, all the related system information, such as the vehicle position and its SOC, is accurately known. This validation approach could be considered as a "best-case" testing, and it is consistent with the test-case-design methods applied to test software, such as boundary value analysis [16] or distributed real time systems [17].

\section{Experiments for model testing and first results}

After defining the CWD model, it is necessary to estimate its capability to determine the quality level assessment for the charging service. The electrical power distribution type that should be supplied at each node is an interesting result from this preliminary stage of CWD development. The traffic and energy results will be reported in the two following sections, and two operational testing scenarios will be analyzed.

\subsection{Parameter setting for the simulated scenarios}

The Reference scenario represents a compatible flow of light vans generated by a logistics centre for multiple deliveries. A second scenario (Alternative) will be explored to analyze how the system performance could be affected by the increase of both the FEV traffic and the minimum allowed technical headway in the CWD lane. In the Alternative scenario, vehicles are generated closer than those in the Reference scenario, but they cannot stay too close while charging, thus creating a delay phenomenon with vehicle platoons in queue.
Table 1 Data related to traffic

\begin{tabular}{ll}
\hline Traffic & \\
\hline Average density for input traffic flow & $10(20) \mathrm{veh} / \mathrm{km} / \mathrm{lane}$ \\
Critical density (at max capacity) & $30 \mathrm{veh} / \mathrm{km} / \mathrm{lane}$ \\
Number of simulated vehicles & $500 \mathrm{veh}$ \\
Coefficient of variation of the headway & 0.3 \\
Minimum traffic headway & $1.5 \mathrm{~s}$
\end{tabular}

Table 2 Data related to infrastructure

\begin{tabular}{ll}
\hline Infrastructure & \\
\hline Total length of the road & $20 \mathrm{~km}$ \\
Average slope & $0 \%$ \\
Sections length & $1 \mathrm{~km}$ \\
Length of the charging zones $\left(L_{\mathrm{CZ}}\right)$ & $20 \mathrm{~m}$ \\
Interdistance $(I)$ & $30 \mathrm{~m}$ \\
Transition coefficient $\left(T_{\mathrm{rk}}\right)$ & 1 \\
System efficiency $\left(\eta_{\mathrm{s}}\right)$ & 0.85 \\
Power per unit of length $\left(P_{\mathrm{CZ}}\right)$ & $50 \mathrm{~kW} / \mathrm{m}$ \\
Minimum headway in CWD lane & $1.5(3) \mathrm{s}$ \\
\hline
\end{tabular}

In Table 1 and Table 2, basic traffic feature data and infrastructure layout parameters are reported for the Reference scenario. The data between brackets indicates the variations introduced in the Alternative scenario.

A critical density value of $30 \mathrm{veh} / \mathrm{km} / \mathrm{lane}$ has been assumed based on the generally adopted values for freeways under basic conditions [18]. Minimum headway values between 1.5 and $2.5 \mathrm{~s}$ have been chosen to consider the use of ADAS [19].

In Table 3, the vehicle data specifies motion performance, energy consumption and energy needs. At each node of the modelled road, the SOC of every vehicle is assessed.

Although some car manufacturers use the currently available adaptive cruise control (ACC) to give the drivers the opportunity to manually choose the minimal headway, they set the absolute minimum headway at $0.9 \mathrm{~s}$ [20]. In this study, a more prudent value of $1.5 \mathrm{~s}$ has been assumed. According to the analysis reported in a previous study [7], a vehicle with a SOC less than $30 \%$ of its target is assumed in an emergency situation (state = "emer") and its desired speed along the CWD lane is set to $30 \mathrm{~km} / \mathrm{h}$; if the charging level is between $30 \%$ and $60 \%$ of the target value, then the vehicle is assumed to be charged in the CWD lane to preserve its SOC (state = "charge") and its desired speed is set to $60 \mathrm{~km} / \mathrm{h}$. Vehicles with a current charge level greater than $60 \%$ of the target SOC are assumed "out" of the CWD lane because they do not need to 
Table 3 Data related to vehicles features

\begin{tabular}{ll}
\hline Vehicles & \\
\hline Average starting SOC & $10 \mathrm{kWh}$ \\
Standard deviation of SOC & 4.5 \\
SOC target & $20 \mathrm{kWh}$ \\
Length of charging the device $\left(L_{\mathrm{CD}}\right)$ & $1 \mathrm{~m}$ \\
SOC limit for "charge" vehicles & $60 \%$ \\
SOC limit for "emer" vehicles & $30 \%$ \\
Desired speed of "charge" vehicles on CWD & $60 \mathrm{~km} / \mathrm{h}$ \\
Desired speed of "emer" vehicles on CWD & $30 \mathrm{~km} / \mathrm{h}$ \\
Max free flow speed on other lanes & $110 \mathrm{~km} / \mathrm{h}$ \\
Average acceleration & $0 \mathrm{~m} / \mathrm{s}^{2}$ \\
Overtake duration & $10 \mathrm{~s}$ \\
Mass (m) & $2500 \mathrm{~kg}$ \\
Cross sectional area $(A)$ & $4.9 \mathrm{~m}{ }^{2}$ \\
$C_{\mathrm{x}}$ & 0.38 \\
$f_{0}$ & $0.12 \mathrm{~m} / \mathrm{s}^{2}$ \\
$f_{2}$ & $0.000005 \mathrm{~m}^{-1}$ \\
Driveline efficiency $\left(\eta_{\mathrm{d}}\right)$ & 0.75 \\
Auxiliary power $\left(P_{\text {aux }}\right)$ & $0.8 \mathrm{~kW}$ \\
\hline
\end{tabular}

recharge. Their speed is then set according to the feasible speed in the other lanes, which depend on the estimated traffic density.

\subsection{Primary traffic results}

In this section, a comparison of selected principal traffic results in the Reference and Alternative scenarios is reported. Because all results depend on the random variables generated at the initial traffic and energy states, multiple replications of this experiment should be examined to observe, using statistical analysis, how the random effects influence the simulation results. However, to better show the traffic and energy performance of the implemented simulation model, through the reading of the calculated variables in identical conditions, the following results will focus on one selected replication that is close to the average value.

The first parameter analyzed is the FEV traffic flow in the CWD lane. Fig. 4 and Fig. 5 compare the traffic flows at the entrance and at the exit of CWD lane, respectively for the Reference and the Alternative scenarios. Fig. 4 shows that in the Reference scenario, the traffic flow in the CWD lane increases along the lane, with concentration phenomena at the exit section, although never reaching the maximum value of $2400 \mathrm{veh} / \mathrm{h}$ related to the minimum technical headway $(1.5 \mathrm{~s})$. In particular, based on the values set for the parameters, an "emer" vehicle increases its SOC and, after reaching the SOC threshold, it increases its

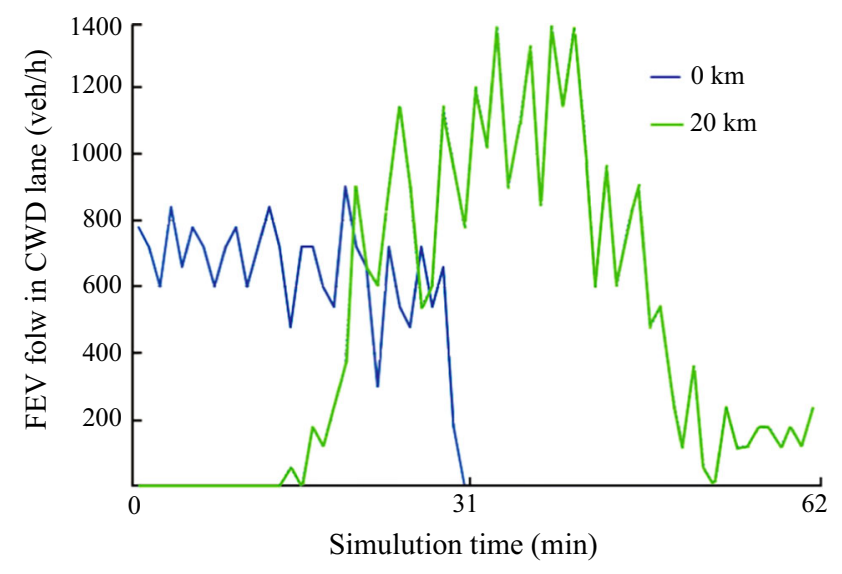

Fig. 4 Traffic flow into the CWD lane at the entrance $(0 \mathrm{~km})$ and the exit node $(20 \mathrm{~km})$ for the Reference scenario

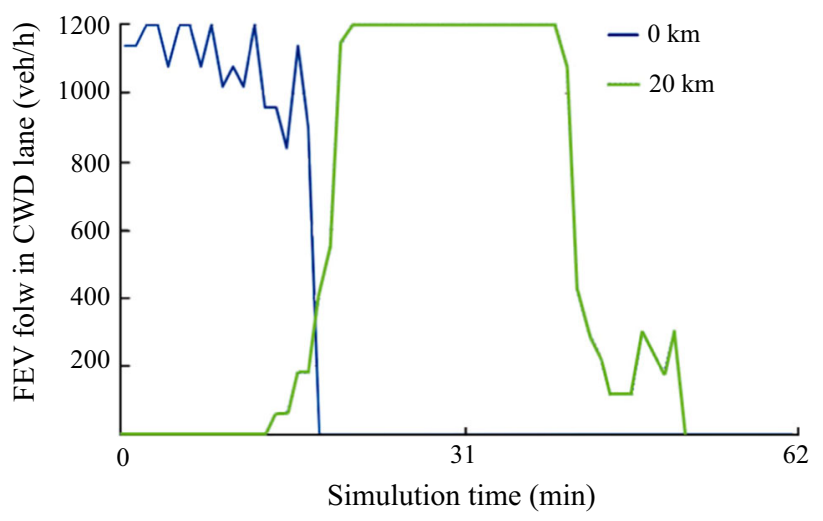

Fig. 5 Traffic flow in the CWD lane at the entrance $(0 \mathrm{~km})$ and the exit node $(20 \mathrm{~km})$ for the Alternative scenario

speed according to the "charge" vehicles desired speed, whereas a "charge" vehicle maintains a constant SOC over time. Consequently, no vehicle leaves the CWD lane, whereas "out" vehicles can enter into the CWD lane during the simulation.

An identical effect can also be observed for the Alternative scenario, in which the higher minimum technical headway value (equal to $3 \mathrm{~s}$ ) defines a lower maximum admissible flow of $1200 \mathrm{veh} / \mathrm{h}$ in the CWD lane. Therefore, traffic conditions at $0 \mathrm{~km}$ approximate the maximum allowable flow. At $20 \mathrm{~km}$, the limit conditions occur for the majority of the simulation time, as illustrated by the plateau in Fig. 5, which is caused by vehicle platoon conditions. In this case, an entrance into the CWD lane or an overtake maneuver may cause a relevant disturbance in the traffic flow, resulting in a sensible reduction in the average speeds of the following vehicles.

Figures 6 and 7, for the Reference and the Alternative scenario, respectively, report the vehicle counts that are detected at each kilometer (at each node), along the CWD 
Time ( $\min )$

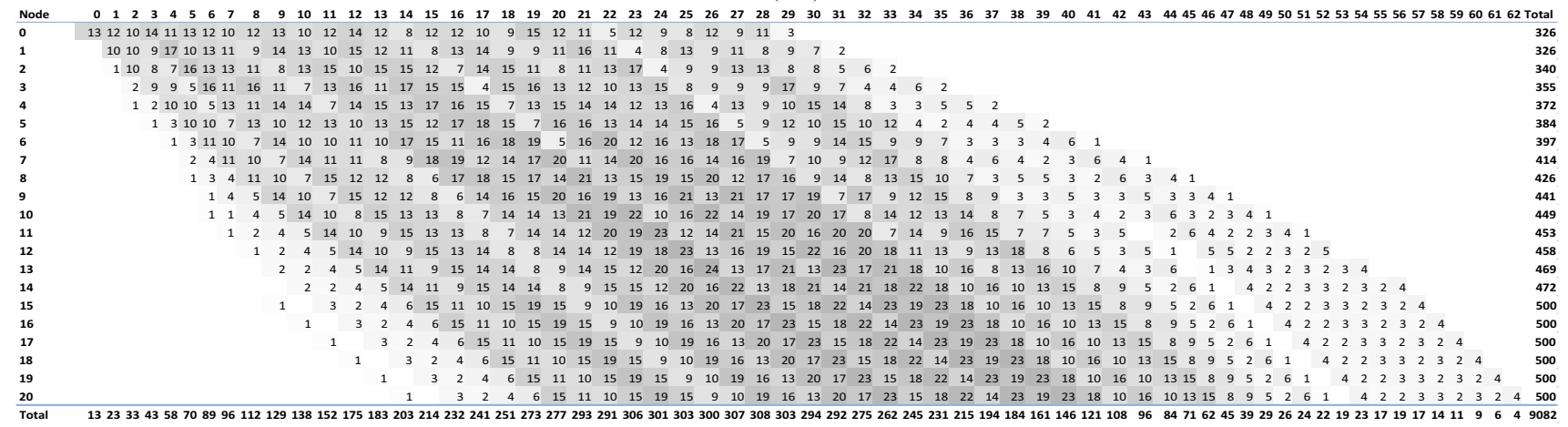

Fig. 6 FEV counts along the CWD lane over time in the Reference scenario

Time ( $\min )$

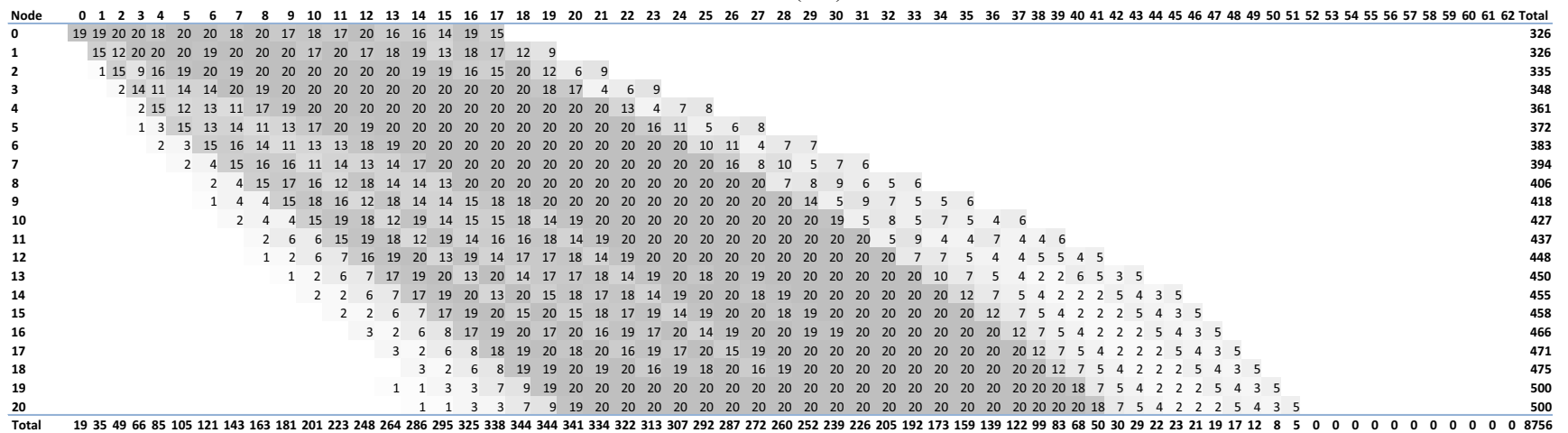

Fig. 7 FEV counts along the CWD lane over time in the Alternative scenario

lane over time. In the grey scale, the higher values are represented with a darker color.

In the Reference scenario, different color areas can be noted, indicating a certain variability of the traffic flow over time. The initial high traffic conditions of the Alternative scenario cause a uniform distribution of the vehicles, highlighted by a flatter coloration. As expected, the CWD lane flow reaches the maximum value allowed by the degraded system of the Alternative scenario (20 veh/min), confirming the previous platoon considerations.

In the Reference scenario, the number of charging vehicles increases more rapidly because the speed in the other lanes is higher as a result of better traffic conditions, thus increasing vehicle energy consumption. Before the final node $(20 \mathrm{~km})$, all generated vehicles must be recharged so they enter the CWD lane.

Because of the battery capacity limitations, all vehicles driving in the unequipped lanes reduce their SOC and reach the "charge" threshold within the last sections. This phenomenon, which is consistent with assumptions, causes the final increase in the vehicle count in the CWD lane.

The second parameter analyzed is the space mean speed. Figures 8 and 9 for the Reference and the Alternative scenario, respectively, report the values on the sections before each node along the CWD lane over time, considering both "charge" and "emer" vehicles. The darker color refers to lower values and therefore the worst traffic condition cases.

The zones in the time-space diagram in which congestion occurs are consistent with the data from the scenarios. Values exceeding the speed limit in the CWD lane $(60 \mathrm{~km} / \mathrm{h})$ are caused by the entries into the CWD lane from the other lanes, where the speeds are higher, because they are related to the established traffic density.

As expected, the lowest speeds for the first sections are presented at the end of the simulation time because only the "emer" and slow vehicles are presented. Any possible "charge" and fast vehicles have previously crossed this section. This concentration of the slower vehicles at the end of the simulation occurs only for the first sections, because after node 14, all "emer" vehicles have increased their SOC over the "charge" threshold, changing their status. In both analyzed scenarios, the average speeds of the traffic flow exceed $30 \mathrm{~km} / \mathrm{h}$.

Finally, delay is the last traffic parameter reported. It is analyzed separately for "charge" and "emer" vehicles. In the Reference scenario, the delay is negligible: considering all simulation time along the CWD lane, it reaches the 


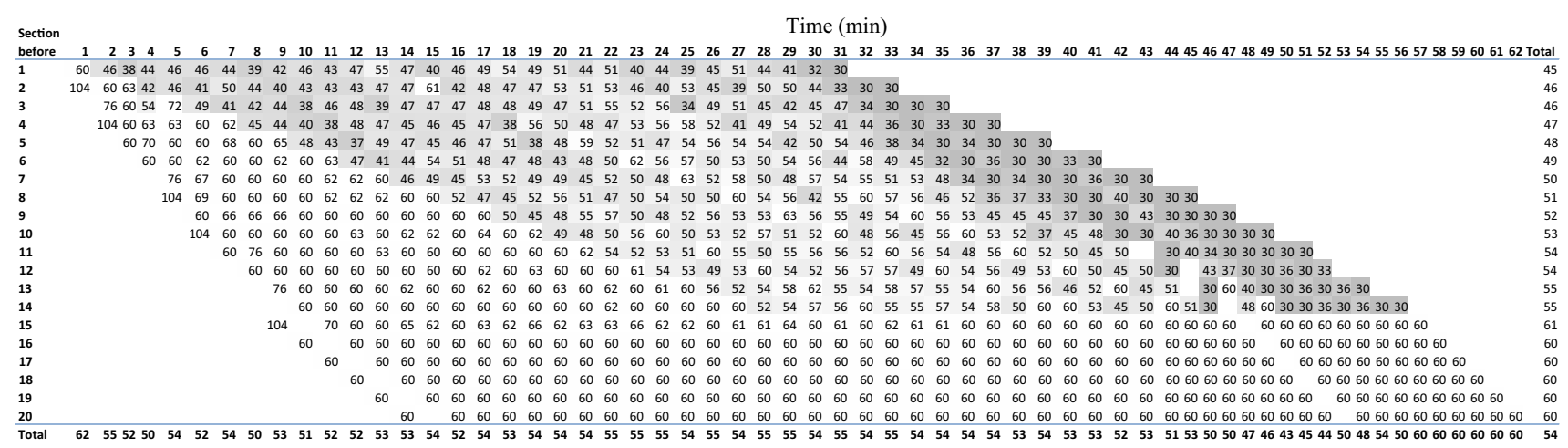

Fig. 8 Space mean speed of FEVs along the CWD lane over time in the Reference scenario

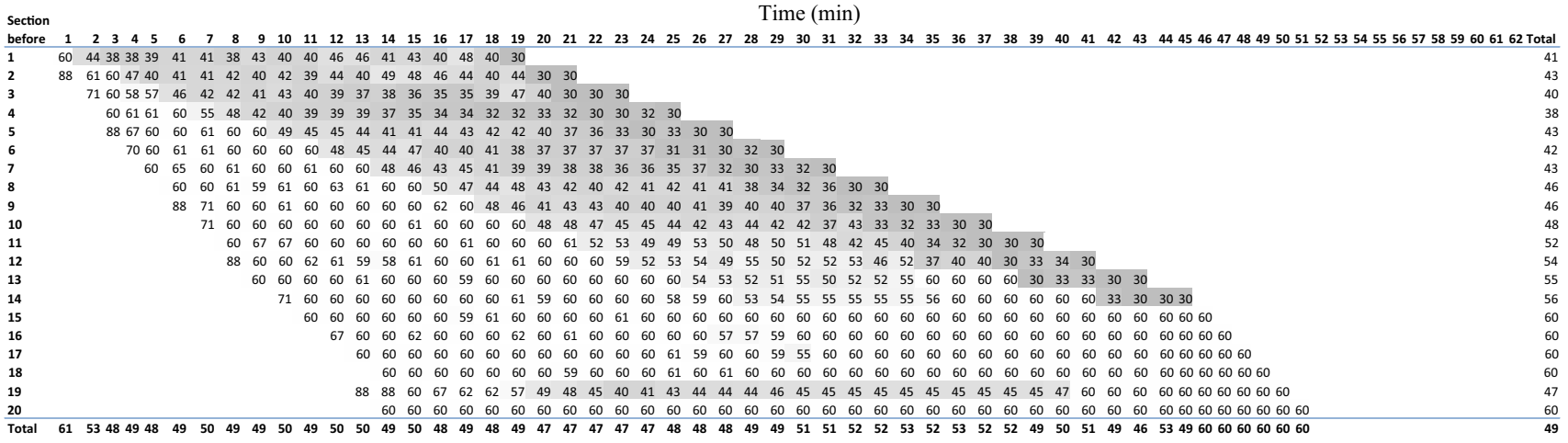

Fig. 9 Space mean speed of FEVs along the CWD lane over time in the Alternative scenario

maximum value of $47 \mathrm{~s}$ for "charge" vehicles at node 13 . This indicates that a delay of $47 \mathrm{~s}$ is assessed by considering all 429 "charge" vehicles in 40 minutes of simulation. Consequently, no space-time table will be reported, which is consistent with the data assumed for this scenario.

The delay for the Alternative scenario is relevant. Figures 10 and 11 report the time-dependent average delay for "charge" and "emer" vehicles over time, respectively, along the CWD lane. The mean delay values are similar for "charge" and "emer" vehicles, between 0 and $45 \mathrm{~s}$ on average. The Alternative scenario traffic conditions generate queues and consequently delay in the traffic flow, causing a decrease of the average speeds. In Fig. 10, the increased delay at the section before node 19 confirms the entry of the last charging vehicles into the CWD lane, where the traffic flow proceeds with vehicle platoons in queue.

\subsection{Energy estimation for CWD}

In this section, selected simulation results related to the CWD energy issues are reported. In Figs. 12 and 13, the energy received at each node by FEVs from the single $\mathrm{CZ}$ placed on the detection point over time are presented for the Reference and Alternative scenarios, respectively. These results confirm that the simulation can describe the CWD energy dynamics. This analysis confirms that the energy required may vary significantly along the road, and it may change over the time. From the grey scale in Fig. 12, multiple waves travelling ahead with an approximate speed of $30 \mathrm{~km} / \mathrm{h}$, which is the speed for emergency vehicles, can be observed for the Reference scenario. The maximum value observed for any $\mathrm{CZ}$ at nodes is $0.3 \mathrm{kWh}$ during one minute; in most cases, it does not continue for more than three consecutive minutes. In the Alternative scenario (Fig. 13) the variation is uniform: for example, the value of $0.4 \mathrm{kWh}$ is constant for longer periods (in some cases, approaching 20 minutes). In this scenario, the higher value of $0.5 \mathrm{kWh}$ was detected for CZs at nodes after $3 \mathrm{~km}, 4 \mathrm{~km}$ and $7 \mathrm{~km}$, but only for few minutes. After reporting the simulation results for the energy required by vehicles along the CWD lane at the selected detection points, a global energy analysis is described here.

Cumulative power profiles can be simulated for the Reference and the Alternative scenarios to estimate the power a single energy provider should supply along the entire CWD system. To obtain complete information about 


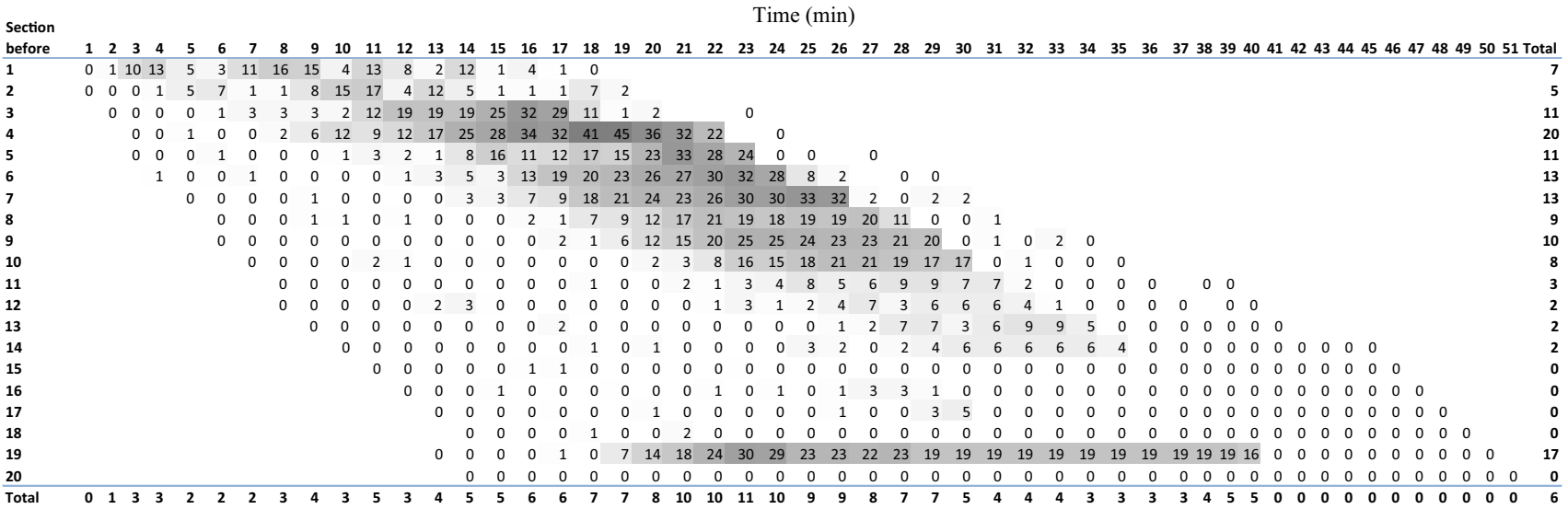

Fig. 10 Average delay for "charge" FEVs along the CWD lane over time in the Alternative scenario

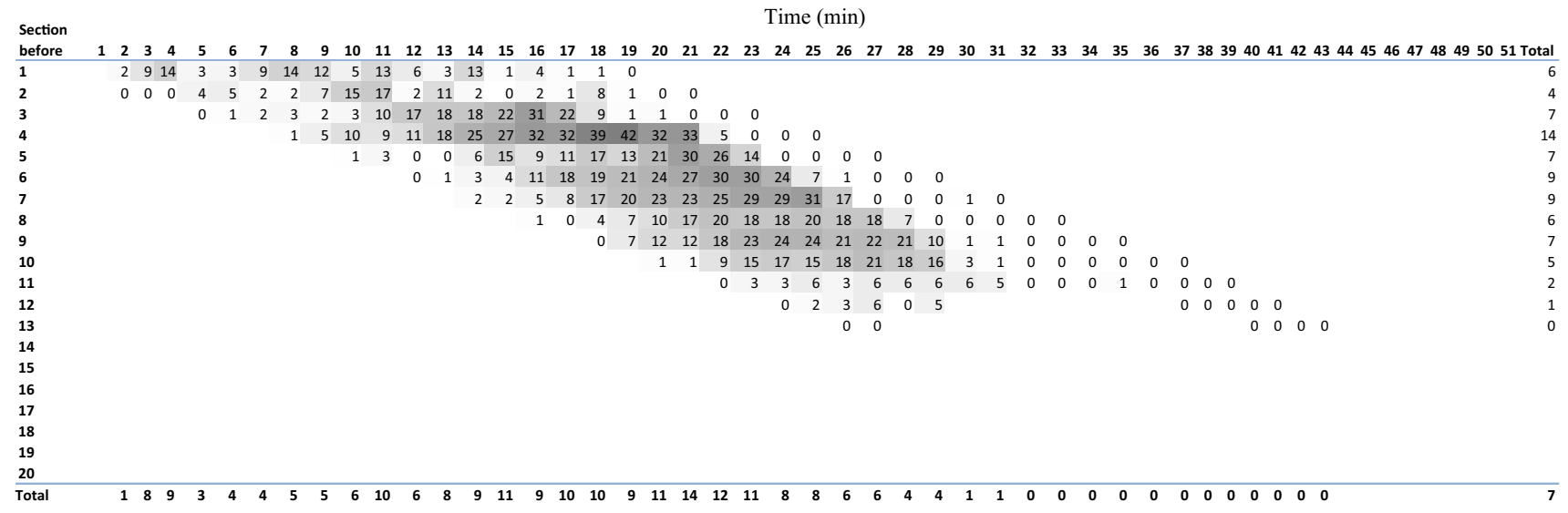

Fig. 11 Average delay for "Emer" FEVs along the CWD lane over time in the Alternative scenario

Time (min)

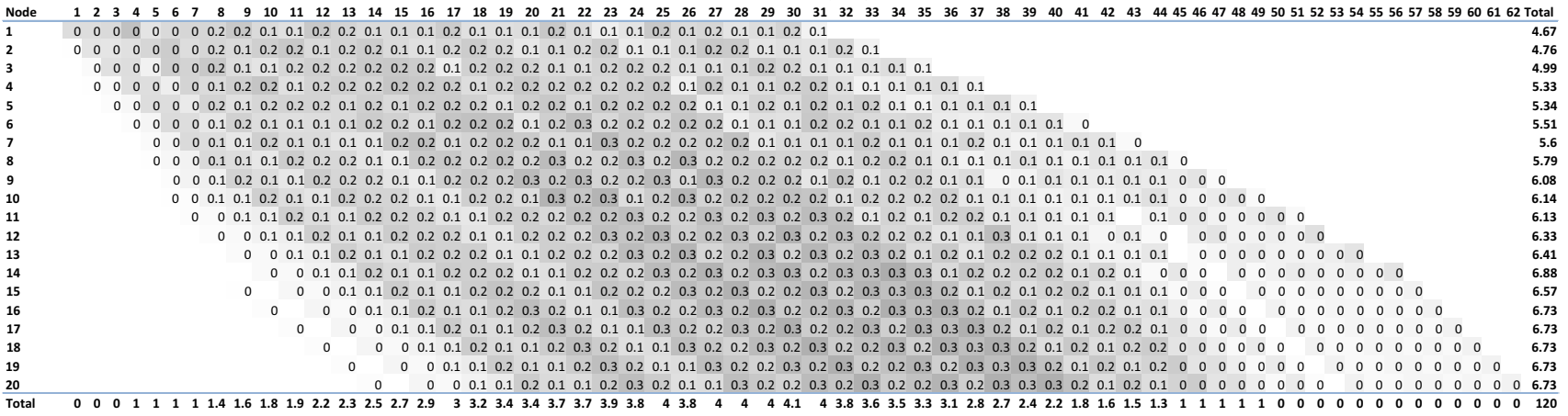

Fig. 12 Energy received (kWh) by FEVs at nodes along the CWD lane over time in the Reference scenario

all of the CZs, a higher resolution of simulation sections is required. Two additional experiments for the Reference and the Alternative scenarios have been performed.

The analyzed nodes were set at a distance of $\mathrm{LCZ}+\mathrm{I}$, equal to $50 \mathrm{~m}$. Fig. 14 and Fig. 15 report the cumulative number of coil on/off switching during the simulation for a $20 \mathrm{~s}$ time widow $(1500 \sim 1520 \mathrm{~s})$ respectively for the
Reference and Alternative scenarios. In the Reference scenario, there is generally a higher occurrence of switching on compared to the Alternative scenario. This result can be confirmed because of the larger number of vehicles in the CWD lane. The variability of the power provided, as estimated by simulation, is evident in the charts in Fig. 14 and Fig. 15 in which the instantaneous 
Time $(\min )$

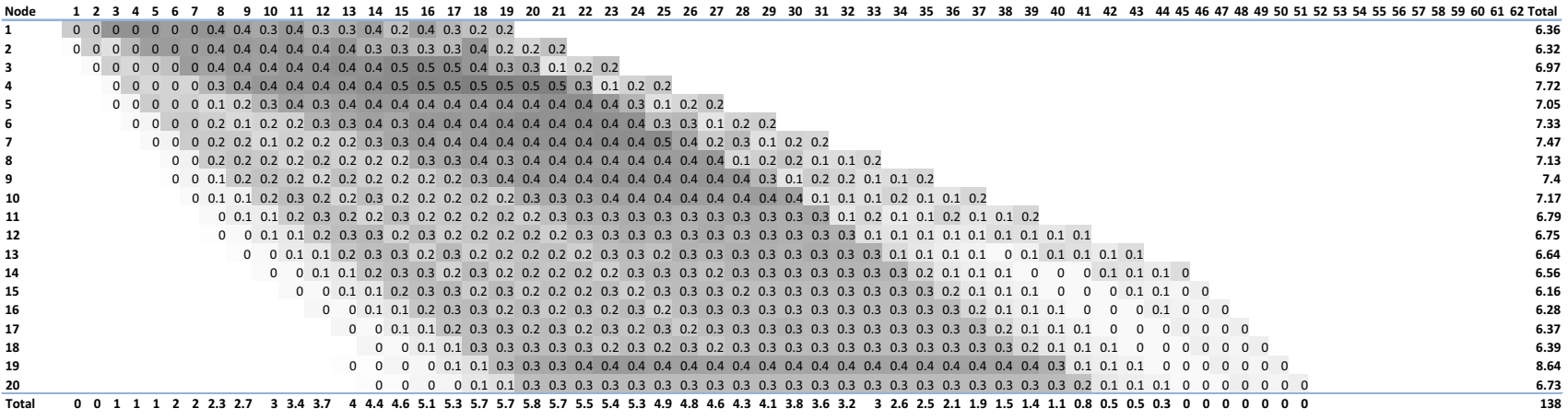

Fig. 13 Energy received (kWh) by FEVs at nodes along the CWD lane over time in the Alternative scenario

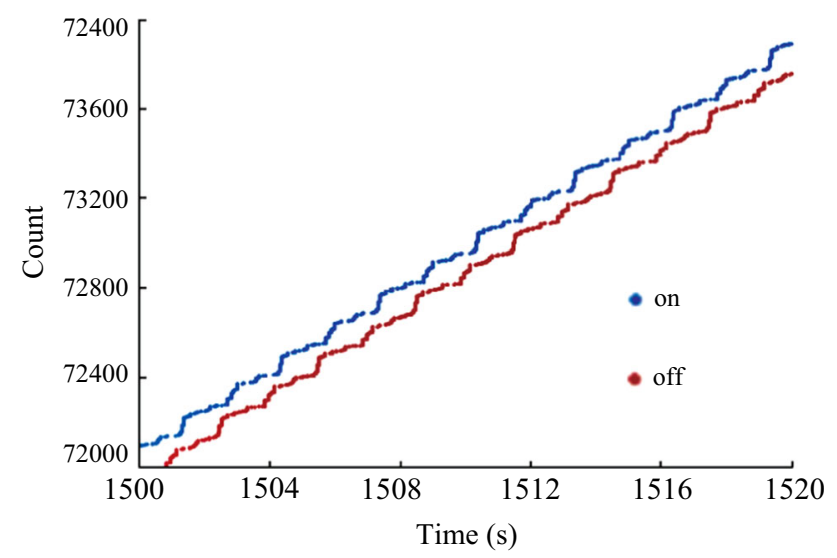

Fig. 14 Cumulative count of on/off switching for all the CZs of the CWD lane during $20 \mathrm{~s}$ for the Reference scenario

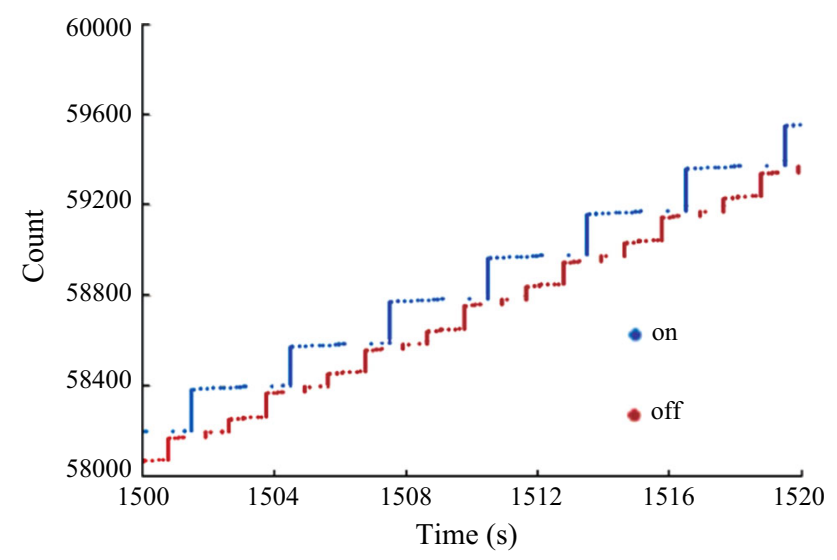

Fig. 15 Cumulative count of on/off switching for all the CZs of the CWD lane during $20 \mathrm{~s}$ for the Alternative scenario

number of CZs in the "ON" state changes in a few seconds for both Reference and Alternative scenarios. The maximum number of CZs simultaneously in the "ON" state is estimated to equal $181 \mathrm{CZs}$ at the simulation time of $1763.2 \mathrm{~s}$ for the Reference scenario and $281 \mathrm{CZs}$ at the

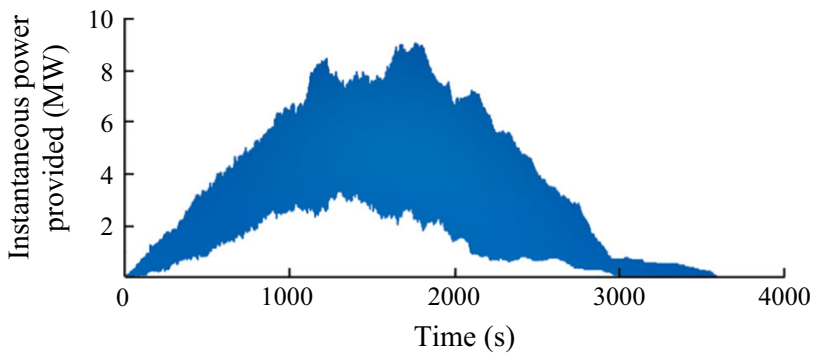

Fig. 16 Instantaneous power provided for the entire $20 \mathrm{~km} \mathrm{CWD}$ lane in the Reference scenario

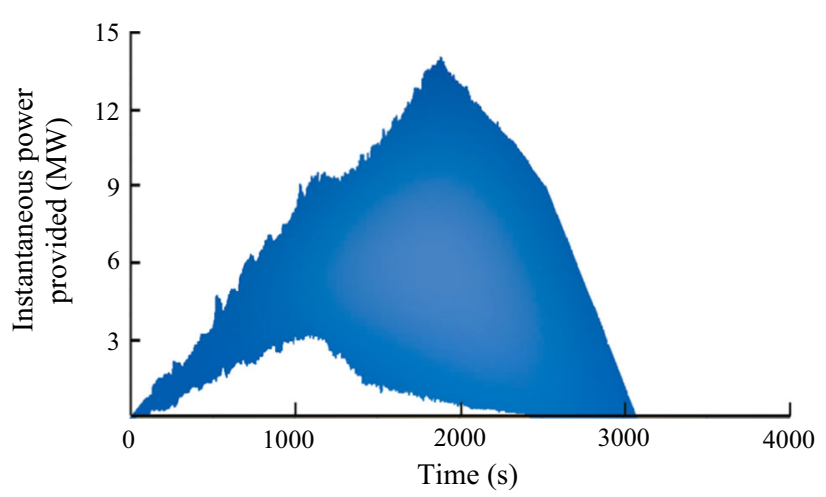

Fig. 17 Instantaneous power provided for the entire $20 \mathrm{~km} \mathrm{CWD}$ lane in the Alternative scenario

simulation time of $1876.5 \mathrm{~s}$ for the Alternative. To better observe the energy variability, the simulated instantaneous power provided for the entire $20 \mathrm{~km} \mathrm{CWD}$ lane is also reported in Fig. 16 and Fig. 17. The minimum and maximum power provided can be clearly identified, by multiplying the number of CZs in the "ON" state by the nominal power provided (Pcz), according to LCD. In addition, a detailed chart of the power provided for the entire CWD lane is presented in Figs. 18 and 19 for an identical $20 \mathrm{~s}$ time window to show the typical pattern for the two simulated scenarios. 


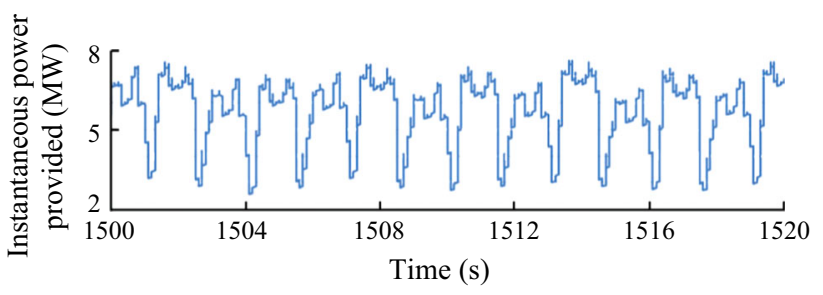

Fig. 18 Instantaneous power provided (MW) for the $20 \mathrm{~s}$ time window for the entire $20 \mathrm{~km} \mathrm{CWD}$ lane in the Reference scenario

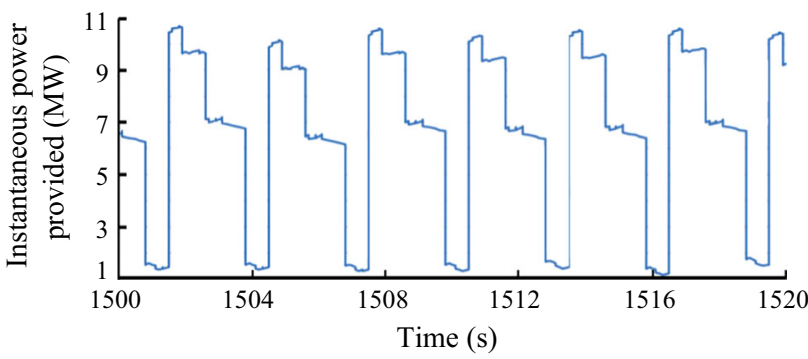

Fig. 19 Instantaneous power provided for the $20 \mathrm{~s}$ time window for the entire $20 \mathrm{~km} \mathrm{CWD}$ lane in the Alternative scenario

\section{Conclusions}

This study presented a method for assessing the performance of the wireless inductive power transfer used to charge electric vehicles while driving. Assuming the CWD system can operate in a scenario with cooperative behavior, the developed traffic model is able to simulate different traffic conditions. Primary traffic parameters can be estimated for the CWD lane, such as the vehicle count and the average speed that are time dependent and change relevantly along the road. This traffic model can manage even intense traffic conditions by simulating vehicle platoons and delays caused by internal traffic interactions (i.e., different vehicle speeds and new entries into the lane) and technical constraints requiring a minimum headway in the CWD lane. Unlike traditional dynamic traffic models, the vehicle motion in this proposal includes the energy needs and charging opportunities because they influence drivers' decisions and then traffic performance. According to their SOC along the road, vehicles are simulated as inside or outside the charging lane, and their speeds are set according to their charging mode. The model has an approximation consistent with the stage of development of CWD technology and the deployment of cooperative driving. Although simplified, it allows for the prediction of many relevant energy issues and possible operational problems.

From the energy point of view, the analyses presented here for a "best case" scenario demonstrates that the traffic also has a relevant effect on the energy that should be supplied by an energy provider. In the Reference scenario simulated, characterized by better traffic conditions, the maximum power that should be supplied for the entire road is approximately $9 \mathrm{MW}$, whereas in the Alternative scenario, in which vehicles proceed slower and are generated closer, the power required by the vehicles on the CWD lane is approximately $14 \mathrm{MW}$. This result is even more relevant considering that the total switching on number is greater in the Reference scenario, thus indicating a major usage of the CWD lane. However, the slower speeds and the platoon conditions require a larger number of coils to be on simultaneously. This critical traffic condition, characterized by platoons with vehicles at a constant distance, generates high peaks in the power trend; in a few tenths of a second, the power required can change by more than $9 \mathrm{MW}$. Generally, the required power trend under platoon conditions is more consistent but with higher peaks.

Acknowledgments This study is partially supported by the eCoFEV project (Grant agreement No. 314411). The authors would like to thank all project partners for their support.

Open Access This article is distributed under the terms of the Creative Commons Attribution License which permits any use, distribution, and reproduction in any medium, provided the original author(s) and the source are credited.

\section{References}

[1] Boulanger AG, Chu AC, Maxx S et al (2011) Vehicle electrification: status and issues. P IEEE 99(6):1116-1138

[2] Kotchapansompote P, Wang YF, Imura T et al (2011) Electric vehicle automatic stop using wireless power transfer antennas. In: Proceedings of the 37th annual conference on IEEE Industrial Electronics Society (IECON'11), Melbourne, Australia, 7-10 Nov 2011, pp 3840-3845

[3] Cassat A, Jufer M (2002) MAGLEV projects technology aspects and choices. IEEE Trans Appl Supercon 12(1):915-925

[4] Stielau OH, Boys JT, Covic GA et al (1999) Battery charging using loosely coupled inductive power transfer. In: Proceedings of the 8th European conference on power electronics and applications (EPE'99), Lausanne, Switzerland, 7-9 Sept 1999

[5] He F, Yin Y, Zhou J (2013) Integrated pricing of roads and electricity enabled by wireless power transfer. Transp Res C-Emer 34:1-15

[6] SLee S, Huh J, Park C et al (2010) On-line electric vehicle using inductive power transfer system. In: Proceedings of the 2010 IEEE energy conversion congress and exposition (ECCE'10), Atlanta, GA, USA, 12-16 Sept 2010, pp 1598-1601

[7] Deflorio F, Guglielmi P, Pinna I et al (2013) Modelling and analysis of wireless "charge while driving" operations for fully electric vehicles. In: Proceedings of the convegno annuale e seminario scientifico della Societ Italiana dei Docenti di Trasporti (SIDT'13), Trieste, Italy, 17-18 Oct 2013

[8] Witold K, Lan L, Yuichi K et al (2014) Deliverable D200.1: Use cases and requirements for an efficient cooperative platform. eCo-FEV, Valbonne

[9] Hoogendoorn SP, Bovy PHL (2001) State-of-the-art of vehicular traffic flow modelling. P I Mech Eng I 215(4):283-303 
[10] Barceló J (2005) Dynamic network simulation with AIMSUN. In: Kitamura R, MasoKuwahara $M$ (eds) Simulation approaches in transportation analysis, part 1. Springer, Boston, pp 57-98

[11] Cascetta E (2001) Transportation systems engineering: theory and methods. Kluwer Academic Publishers, Dordrecht

[12] Ben-Akiva ME, Gao S, Wei Z et al (2012) A dynamic traffic assignment model for highly congested urban networks. Transp Res C-Emer 24:62-82

[13] Deflorio F, Castello L (2014) Traffic modeling of a cooperative charge while driving system in a freight transport scenario. In: Proceedings of the 4th international symposium of transport simulation and international workshop on traffic data collection and ITS standardisation (ISTS \& IWTDCS'14), Ajaccio, France, 1-4 June 2014, pp 7-18

[14] SS-EN 14725:2004. Space engineering - Verification. 2004

[15] Haskins C (2011) Systems engineering handbook V. 3.2.2. INCOSE-TP-2003-002-03.2.2. The International Council on Systems Engineering (INCOSE), San Diego, CA, USA

[16] Myers GJ, Badgett T, Sandler C (2004) The art of software testing. Wiley, New York

[17] Gutiérrez JCP, Gutierrez Garcia JJ, Gonzalez Harbour M (1998) Best-case analysis for improving the worst-case schedulability test for distributed hard real-time systems. In: Proceedings of the 10th Euromicro workshop on real-time systems, Berlin, Germany, 17-19 June 1998, pp 35-44

[18] Daganzo CF (1997) Fundamentals of transportation and traffic operations. Pergamon, Amsterdam

[19] Yannis G, Golias J, Antoniu C (2004) Combining traffic simulation and driving simulator analyses for advanced cruise control system impact identification. In: Proceedings of the 83rd annual meeting of the transportation research board, Washington, DC,USA, 11-15 Jan 2004
[20] Hegeman G, Brookhuis K, Hoogendoorn S (2005) Opportunities of advanced driver assistance systems towards overtaking. Eur J Transp Infrastruct Res 5(4):281-296

Francesco Paolo DEFLORIO received the Ph.D. in Automatics and Information Science in Transportation Systems from Politecnico di Torino, Italy, where he is assistant professor in Transport System Engineering since 2006. His research interests include modeling and applications of traffic and transportation systems, such as dynamic route guidance in road networks, traffic and energy simulation analysis, intelligent transport systems (ITS).

Luca CASTELLO received the M.Sc. in Civil Engineering from Politecnico di Torino, Italy, in 2011, where he worked as a research fellow from 2013 to 2014. Currently, he is working as a consultant for the transportation research center of IVECO. His research interests include traffic simulations and new vehicle technologies, primarily concerning vehicle energy needs and emissions.

Ivano PINNA received the M.Sc. in Mechanical Engineering from Politecnico di Torino, Italy, where he is research contractor and $\mathrm{Ph} . \mathrm{D}$ student. His research interests include intelligent transport systems, road safety, ADAS, electric and hybrid vehicles, "charge while driving" analysis from transport systems viewpoint.

Paolo GUGLIELMI received the Ph.D. degree in Electrical Engineering from the Politecnico di Torino, Turin, Italy, in 2001. In 1997, he joined the Department of Electrical Engineering, Politecnico di Torino as Research Assistant. Since 2012, he is Associate Professor at the same university. His research interests include power electronics for wireless power transfer, high-performance drives, and computeraided design of electrical machines. 